\title{
Numerical Investigation of the Stability and Spintronic Properties of Selected Quaternary Alloys
}

\author{
Adewumi I. Popoola and S. Babatunde Akinpelu
}

\section{ABSTRACT}

\begin{abstract}
The use of electronic charge and spins (spintronics) has been proposed for much better data storage. This class of material is believed to have excellent capability for data integrity, low dynamic power consumption and highdensity storage that showcases excellent protection against data loss. The spintronic and related properties have been investigated on four newly proposed quaternary alloys (NbRhGeCo, NbRhGeCr, NbRhGeFe and NbRhGeNi) through the first-principles calculation method of the Density Functional Theory (DFT). Specifically, the phonon frequencies, elastic stabilities, and the electronic structure were systematically studied in the full Heusler structure. The results predict that NbRhGeFe and NbRhGeCr are elastically and structurally stable. Both NbRhGeFe and NbRhGeCo are halfmetals with ferromagnetic character, but NbRhGeCo is unfortunately elastically unstable. NbRhGeCr and $\mathrm{NbRhGeNi}$ are non-magnetic metallic alloys in their spin channels. All the results predict NbRhGeFe to be the only suitable among all the four alloys for spintronic application.
\end{abstract}

Keywords: Exchange-splitting, Ferromagnetism, Polarization ratio,
Published Online: July 08, 2021

ISSN: $2684-4451$

DOI : $10.24018 /$ ejphysics.2021.3.4.86

A. I Popoola*

Federal University of Technology, Akure. Nigeria.

(e-mail: aipopoola@futa.edu.ng or

ispopoola71@gmai.com)

\section{S. B. Akinpelu}

Federal University of Technology, Akure. Nigeria.

(e-mail: atunde5@gmail.com)

*Corresponding Author

\section{INTRODUCTION}

The world has witnessed digital revolution (robotics, artificial intelligence, machine learning etc.). This has led to innovations, such as the robotic waiters, driver-less taxis, ecommerce, and tele-conferencing/medicine, which will continue to increase productivity. In all of these, data acquisition, storage, and transmission - both in size and integrity are cardinal to achieving the various objectives of modern-day technology. The traditional Non-Volatile Memories (NVMs) such as the Compact Disk, which can be Read or Written into (CD-R/W) and Hard Disk Drives (HDDs) uses magnetic material and retain their content even when their electric power is switched off. The NVMs are very sensitive to external magnetic fields and thermal fluctuations [1], [2] in which their data are easily lost when intentionally or accidentally exposed. It is also difficult to achieve higher storage density and capabilities with traditional NVMs, because applications using them do suffer from high static power caused by intrinsic current leakage and dynamic power consumption due to long interconnection delays [3]. The search for a better storage media led to the use of spin and charge (spintronics) for data storage. The technology for spintronic applications has created many nano devices, including spin valves and the Magnetic Tunnel Junction (MTJ) valve which has increased the memory capacity of HDDs more than 1000 times in the last ten years [4], [5]. In terms of speed, non-volatility and high data density, spintronic devices are very attractive. The Spin Transfer Torque Magnetic Random Access Memory (STT-MRAM) as an example can allow power-off in the standby state [6]. Spintronic memory devices are able to reduce dynamic power loss while providing nearly infinite endurance which is required in high density and repeated-access memory medium.

The spintronic phenomenon was first described around 1988 through the Giant Magneto-resistance effect (GMR). The spin valve, which is the active element in the read head of HDD storage device use the GMR effect. Following the discovery of GMR, other technologies that utilizes the spintronic effect includes the Tunnel Magneto Resistance (TMR) and the Magnetic Tunnel Junction (MTJ), which has been in use for developing Magnetic Random aAcess Memory (MRAM) devices. The category of materials already proposed for spintronic applications are majorly magnetic materials [7]-[11], topological insulators [12], [13] and magnetic semiconductors [14]-[20]. The development of the spin Field Effect Transistor had showed that spintronic devices can only be realized with ferromagnetic semiconductors or metals [21]. For the sake of high Curie temperature and diverse magnetic phenomenon, materials with the Heusler structure have been shown to be very effective for formulating spintronic devices [22]-[24].

In a recent study, $\mathrm{NbRhGe}$ was predicted to be elastically and structurally stable with an indirect energy bandgap [25]. Given its optimum mechanical properties, it is the intention of the present study to predict new properties - particularly those relevant to data storage using NbRhGe as the base material. Therefore, magnetic elements are added into NbRhGe using the full Heusler structure. The selected elements are nickel (Ni), chromium $(\mathrm{Cr})$, cobalt $(\mathrm{Co})$ and iron (Fe). They are transitions metals belonging in the d-block of the periodic table. Their electronic structure are: $\mathrm{Ni}=$ 
$\left(3 d^{8} 4 s^{2}\right), \mathrm{Cr}=\left(3 d^{5} 4 s^{1}\right), \mathrm{Fe}=\left(3 d^{6} 4 s^{2}\right)$ and $\mathrm{Co}=$ $\left(3 d^{7} 4 s^{2}\right)$ respectively. Among other things, the elastic as well as the dynamic stability of each composition are investigated. The overview of the methodology used in the computation is given in section 2 . The results of the calculation alongside relevant discussion are given in section 3. The work done is finally summarized in section 4 .

\section{Computational Details}

The crystal structure used for simulating $\mathrm{NbRhGeX}(\mathrm{X}=$ $\mathrm{Cr}, \mathrm{Co}, \mathrm{Fe}, \mathrm{Ni}$ ) is shown in Fig.1. It is a face centred cubic structure (space group $=\mathrm{F} 43 \mathrm{~m}$ ). Its Wyckoff atomic coordinates are $4 \mathrm{a}(0,0,0) 4 \mathrm{~b}(1 / 2,1 / 2,1 / 2), 4 \mathrm{c}(1 / 4,1 / 4,1 / 4)$ and $4 \mathrm{~d}(3 / 4,3 / 4,3 / 4)$. The structure can take maximum of four elements, where the elements can come from same group or different element groups. All the calculations were performed with the Quantum Espresso ab initio code [26]. The generalized gradient approximations [28], was used to treat the electron exchange correlation potential within the KohnSham equation of DFT [27]. Acceptable results were obtained when integration over the Brillouin-zone was done using shifted k-points of $1000(10 \times 10 \times 10)$ according to the Monkhorst-Pack scheme [29]. The criterion for energy convergence was $10^{-4} \mathrm{Ry}$. The first task carried out was geometry optimization, where the potential energy surface was sampled repeatedly until minima forces on the atoms were obtained. Each alloy was optimized for the nonmagnetic, ferromagnetic and antiferromagnetic states. After obtaining the ground state structure, the formation $\left(E^{F}\right)$ and cohesive $\left(E^{C}\right)$ energies were evaluated using (1) and (2).

$$
\begin{aligned}
& E^{F}=E_{t}^{\mathrm{X}^{b}}-E_{t}^{A^{b}}-E_{t}^{B^{b}}-E_{t}^{C^{b}}-E_{t}^{D^{b}} \\
& E^{C}=E_{t}^{\mathrm{X}^{b}}-E_{t}^{A^{a t}}-E_{t}^{B^{a t}}-E_{t}^{C^{a t}}-E_{t}^{D^{a t}}
\end{aligned}
$$

where $E_{t}^{\mathrm{X}^{b}}$ is the bulk ground state energy for $\operatorname{NbRhGeX}(\mathrm{X}=$ $\mathrm{Cr}, \mathrm{Co}, \mathrm{Fe}, \mathrm{Ni}$ ) while $E_{t}^{A^{b}}$ through $E_{t}^{D^{b}}$ and $E_{t}^{A^{a t}}$ through $E_{t}^{D^{a t}}$ are the ground state energy for the participating elements $(\mathrm{Nb}$, $\mathrm{Rh}, \mathrm{Ge}, \mathrm{Fe} / \mathrm{Cr} / \mathrm{Co} / \mathrm{Ni}$ ) in their bulk states and the energies of each isolated atoms in their respective structures.

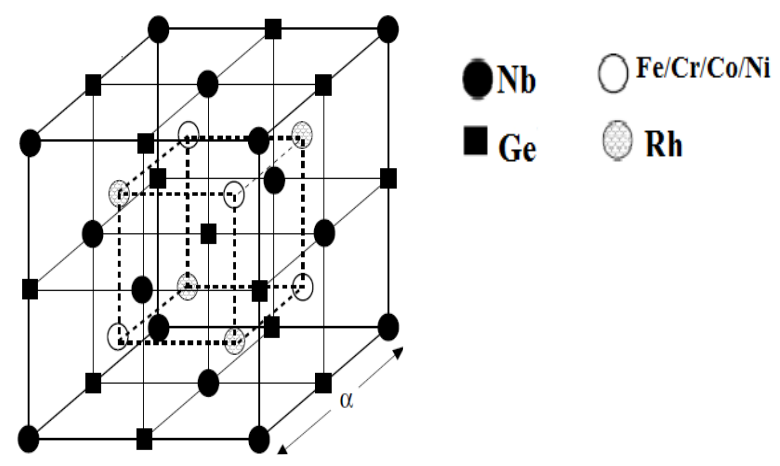

Fig. 1. Illustration of the full-Heusler $\left(\mathrm{L} 2{ }_{1}\right)$ structure. The site preference for $\mathrm{Nb}, \mathrm{Rh}, \mathrm{Ge}$ and $\mathrm{Co} / \mathrm{Ni} / \mathrm{Cr} / \mathrm{Fe}$ are given in the legend.

The ground state lattice constant and bulk modulus were calculated using the Murnaghan's equation of state [30]. Being cubic, there are three elastic constants $\left(C_{11}, C_{12}, C_{44}\right)$ that fully describes the mechanical properties of $\mathrm{NbRhGeX}$ $(\mathrm{X}=\mathrm{Cr}, \mathrm{Co}, \mathrm{Fe}, \mathrm{Ni}$ ). The procedures followed to obtain these elastic constants are as follows [31]. A strain was applied to the crystal and the energy versus the strain was measured, with the elastic constant determined from the curvature of this function at zero strain. Two types of lattice strain were applied - a volume-conserving orthorhombic strain:

$$
\varepsilon=\left(\begin{array}{ccc}
x & 0 & 0 \\
0 & -x & 0 \\
0 & 0 & x^{2} / 1-x^{2}
\end{array}\right)
$$

whose total energy is an even function of distortion $x$ :

$$
\Delta E(x)=\Delta E(-x)=V\left(C_{11}-C_{12}\right) x^{2}+\theta\left[x^{4}\right]
$$

and a monoclinic volume-conserving strain:

$\varepsilon=\left(\begin{array}{ccc}0 & \frac{x}{2} & 0 \\ x / 2 & 0 & 0 \\ 0 & 0 & x^{2} / 4-x^{2}\end{array}\right)$

whose total energy dependence is of the form:

$\Delta E(x)=\Delta E(-x)=\frac{1}{2} V C_{44} x^{2}+\theta\left[x^{4}\right]$

The value of the distortion $x$, was varied from zero (for the equilibrium state) to $\pm 5 \%$. The elastic constant values are obtained from the derivatives of $\Delta E$ with respect to $x^{2}$. After obtaining the single-crystal elastic constants, the related properties such as the bulk modulus can be evaluated. The Hill average for polycrystal moduli [32] is considered better in terms of accuracy and the expression for the bulk modulus for a cubic crystal is:

$B=C_{11}+2 C_{12} / 3$

Other properties that can be evaluated from the elastic constants includes the shear $(G)$ and Young's modulus $(E)$, Frantsevich's ratio $(G / B)$, Zener anisotropy factor $(A)$ [33], Kleinman internal-displacement parameter $(\zeta)$ [34], Poisson's ratio $(v)$, sound velocity $\left(v_{\mathrm{m}}\right)$ and the Debye temperature $\left(\Theta_{\mathrm{D}}\right)[35]$ as well as the Vicker's hardness index $\left(H_{\mathrm{v}}\right)$ [36]. The expressions for these elastic constant dependent properties are given in (8) through (16).

$$
\begin{aligned}
& G_{R}=5\left(C_{11}-C_{12}\right) C_{44} /\left[4 C_{44}+3\left(C_{11}-C_{12}\right)\right] ; \\
& G_{V}=C_{11}-C_{12}+3 C_{44} / 5
\end{aligned}
$$

where

$$
G=G_{R}+G_{V} / 2
$$

$E=9 B G / 3 B+G$

$$
v=3 B-2 G / 2(3 B+G)
$$


$C^{\prime}=C_{12}-C_{44}$

$A=2 C_{44} / C_{11}-C_{12}$

$\zeta=C_{11}+8 C_{12} / 7 C_{11}+2 C_{12}$,

$H_{V}=2\left(G^{3} / B\right)^{0.585}-3$,

$v_{l}=\sqrt{1 / 3 \rho[3 B+4 G]} ; \quad v_{t}=\sqrt{G / \rho} ; \quad v_{m}=\left[1 / 3\left(2 / v_{t}^{3}+\right.\right.$ $\left.\left.1 / v_{l}^{3}\right)\right]^{-1 / 3}$;

$$
\Theta_{D}=\hbar / k_{B}\left[3 n / 4 \pi\left(N_{A} \rho / M\right)\right]^{1 / 3} v_{m}
$$

where the Debye temperature $\left(\Theta_{D}\right)$ is a function of the Boltzmann constant $\left(k_{B}\right)$, the Plank constant $(\hbar)$, the number of atoms per formula unit $(n)$, the Avogadro constant $\left(N_{A}\right), \rho$ is the density of the material and $M$ is the molecular weight.

The phonon frequencies were calculated as a second-order derivative of the total energy with respect to atomic displacements using the Density Functional Perturbation Theory (DFPT) [37].

\section{RESULTS AND DiSCUSSION}

\section{A. Ground-State Structure, Lattice Constants, Formation/Cohesive Energies and Elastic Constants}

The ground state structures of $\mathrm{NbRhGeFe}$ and $\mathrm{NbRhGeCo}$ had lowest energy for the ferromagnetic state while $\mathrm{NbRhGeNi}$ and $\mathrm{NbRhGeCr}$ had their lowest energy for the non-magnetic state. At the zero-energy level (ground state), $\mathrm{Nb}$ preferred the $4 \mathrm{a}(0,0,0)$ position, while Ge occupied the $4 \mathrm{~b}(1 / 2,1 / 2,1 / 2)$ position. The Rh element locates in the $4 \mathrm{c}(1 / 4$, $1 / 4,1 / 4)$ position, with the $4 \mathrm{~d}$ site $(3 / 4,3 / 4,3 / 4)$ occupied by $\mathrm{Fe} / \mathrm{Cr} / \mathrm{Co} / \mathrm{Ni}$ respectively. The lattice constants $\left(L^{C}\right)$, formation/cohesive $\left(E^{F} / E^{C}\right)$ energies and the elastic constants as well as the related mechanical properties are given in Table I. A negative value for $E^{F}$ is an indication of a thermodynamically feasible composition. The cohesive energies are negative and large enough, predicting strong bonding forces in the compounds. Any material for engineering purpose must have mechanical stability. From the elastic constants, the stability criteria for cubic lattices according to Born [38] are: $C_{11}-C_{12}>0, C_{11}>0, C_{44}>0$ and $\left(C_{11}+2 C_{12}\right)>0$. The results in Table 1 predict that $\mathrm{NbRhGeFe}$ and $\mathrm{NbRhGeCr}$ are mechanically stable while $\mathrm{NbRhGeCo}$ and $\mathrm{NbRhGeNi}$ are not stable.

The ductility or brittleness of a material plays key role in its application. Brittle material is not malleable while ductile materials are malleable and can accommodation a lot of deformation before it is fractured. The Pugh and Frantsevich ratios provide easy ways to theoretically evaluate brittleness or ductility in a material. When the Pugh ratio $(B / G)$ is $\geq 1.75$ such material is ductile, else it is brittle [39]. On the other hand, a material is considered to be ductile if its Frantsevich's ratio $(G / B)$ is $\leq 1.06$, else it is brittle [40]. Therefore, the results in Table 1 predicts that $\mathrm{NbRhGeFe}$ and $\mathrm{NbRhGeCr}$ are ductile alloys $(\mathrm{B} / \mathrm{G}>1.75$ and $\mathrm{G} / \mathrm{B}<1.06)$ at ambient conditions while $\mathrm{NbRhGeNi}$ and $\mathrm{NbRhGeCo}$ are not mechanically stable. The Cauchy pressure $\left(C_{p}\right)$ is another reliable way of checking for ductility or brittleness in a material [41]. A negative value for $C_{p}$ would indicate a brittle material, else it is ductile. The $C_{p}$ result further predicts that $\mathrm{NbRhGeFe}$ and $\mathrm{NbRhGeCr}$ should be ductile in alloys. The Kleinman parameter $(\zeta)$ is basically used to determine the ease of bond bending to bond stretching. With $\zeta=0$, bond stretching is more favored is the material than bending while bond bending occurs more than stretching when $\zeta=1$ [42]. The $\zeta$ results, predicts higher percentage of bonding stretching in the alloys. This further confirmed that $\mathrm{NbRhGeFe}$ and $\mathrm{NbRhGeCr}$ should be ductile alloys.

The hardness test for $\mathrm{NbRhGeX}(\mathrm{X}=\mathrm{Fe}, \mathrm{Co}, \mathrm{Ni}, \mathrm{Cr})$ was theoretically done using the Vicker's hardness $\left(H_{\mathrm{V}}\right)$ calculation method. A material is considered hard if its Vicker hardness is $\geq 40 \mathrm{GPa}$ [43]. According to the results in Table I, NbRhGeFe and $\mathrm{NbRhGeCr}$ are predicted to be extremely hard materials while $H_{\mathrm{V}}$ for $\mathrm{NbRhGeNi}$ and $\mathrm{NbRhGeCo}$ cannot be determined due to their mechanical instability.

TABle 1: Calculated Isotropic Bulk Modulus B, Shear Modulus G, Young's Modulus E, Frantsevich's Ratio (G/B), Elastic Constants

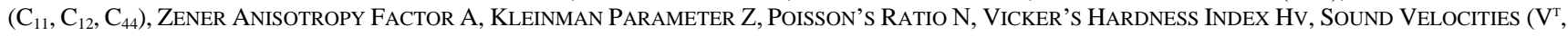

\begin{tabular}{ccccc}
\multicolumn{4}{c}{$\left.\mathrm{V}_{\mathrm{L}}, \mathrm{V}_{\mathrm{M}}\right)$, AND THE DEBYE TEMPERATURE $\Theta \mathrm{D}$, FOR NBRHGEX $(\mathrm{X}=\mathrm{CR}, \mathrm{CO}, \mathrm{Fe}, \mathrm{Ni})$ ALLOYS } \\
\hline Calculated & NbRhGeCr & NbRhGeFe & NbRhGeNi & NbRhGeCo \\
\hline$L^{C}(\AA)$ & 6.140 & 6.129 & 6.139 & 6.136 \\
$E^{c}(\mathrm{eV})$ & -364.012 & -457.192 & -441.613 & -440.102 \\
$E^{F}(\mathrm{eV})$ & -0.798 & -1.426 & -1.374 & -1.448 \\
$B(\mathrm{GPa})$ & 203.513 & 198.939 & 195.1222 & 194.5660 \\
$B_{0}(\mathrm{GPa})$ & 203.820 & 201.550 & 202.579 & 196.782 \\
$G_{(\mathrm{GPa})}$ & 58.609 & 16.282 & -67.626 & -0.263 \\
Frantsevich's ratio $(G / B)$ & 0.289 & 0.081 & -0.345 & -0.00135 \\
Pugh $(\mathrm{B} / G)$ & 3.465 & 46.463 & -2.895 & -741.741 \\
$E(\mathrm{GPa})$ & 160.467 & 47.537 & -228.847 & -21.427 \\
$C_{11}(\mathrm{GPa})$ & 273.959 & 199.395 & 120.579 & 128.752 \\
$C_{12}(\mathrm{GPa})$ & 168.745 & 198.597 & 232.394 & 2281.090 \\
$C_{44}(\mathrm{GPa})$ & 62.988 & 52.350 & -66.301 & 7.802 \\
$A$ & 0.004 & 0.939 & 1.185 & -0.007 \\
$\zeta$ & 0.720 & 0.997 & 1.512 & 3.363 \\
$v$ & 0.369 & 0.427 & 0.677 & 0.569 \\
$H_{\mathrm{v}}(\mathrm{GPa})$ & 84.283 & 177.698 & ----- & ----- \\
$v_{t}(\mathrm{~m} / \mathrm{s})$ & 2497.566 & 1329.691 & ---- & ---- \\
$v_{l}(\mathrm{~m} / \mathrm{s})$ & 5478.104 & 4894.657 & 3464.292 & 11701.070 \\
$v_{m}(\mathrm{~m} / \mathrm{s})$ & 2792.945 & 1505.992 & ---- & ---- \\
$\Theta_{\mathrm{D}}(\mathrm{K})$ & 347.562 & 182.069 & ----- & 24.650 \\
$C_{\mathrm{p}}(\mathrm{GPa})$ & 105.757 & 146.247 & 298.694 & 2273.288 \\
$\mu_{B}$ & 0.01 & 1.63 & 0.00 & 1.48 \\
\hline & & & &
\end{tabular}


Materials that are required to take complex shapes during engineering processes (such as data storage devices) need to have high bonding strength [44], [45]. The bulk modulus $(B)$, which measures the resistance of a material to external deformation, together with the materials resistance to shape change via shearing forces (i.e., the shear modulus, $G$ ) and the degree of stiffness of the material (i.e., the Young's modulus $Y$ ) can together be used to understand the bonding strength of a material [46]-[49]. When the results for $B, G, Y$ are taken together with the already discussed $H_{\mathrm{V}}, C_{\mathrm{p}}$ or G/B then one can conclude that $\mathrm{NbRhGeFe}$ and $\mathrm{NbRhGeCr}$ are strong alloys with expected high yield stress. Incidentally, the equation of states derived bulk modulus $\left(B_{0}\right)$ and the one obtained from the elastic constants $(B)$ are only apart by about $1.31 \%$ for $\mathrm{NbRhGeFe}$ and merely $0.15 \%$ for $\mathrm{NbRhGeCr}$. This is a testimony to the high degree of self-consistency of the present calculation.

The relationship between the transverse and longitudinal strains along the elastic loading direction is called the Poisson's ratio $(v)$. The type of bond existing in a material can be gauged, using the Poisson ratio. If $v \approx 0.1$, covalent bonding is predicted, if $v \approx 0.23$ it is ionic and the bonding is metallic if $v \approx 0.33$ [50], [51]. The $v$ results in Table I, predicts that $\mathrm{NbRhGeFe}$ and $\mathrm{NbRhGeCr}$ should exhibit metallic bond.

\section{B. Electronic Structure and Magnetic Properties}

The calculated total magnetization $\left(\boldsymbol{\mu}_{\boldsymbol{B}}\right)$ for $\mathrm{NbRhGeFe}$, $\mathrm{NbRhGeCr}, \mathrm{NbRhGeCo}$ and $\mathrm{NbRhGeNi}$ are given in Table 1. NbRhGeFe $\left(\mu_{B}=1.6\right)$ and NbRhGeCo $\left(\mu_{B}=1.4\right)$ can be predicted to be magnetic due to their positive magnetic moments. The Density of States (DoS) result is given in Fig.
2 (a) to (d). A very strong exchange splitting can be seen (characteristic of a ferromagnet) at the Fermi level $\left(E_{F}\right)$ between the up-spin channel and the down-spin channel of $\mathrm{NbRhGeFe}$ and NbRhGeCo. Because NbRhGeCr and NbRhGeNi are non-magnetic, their DoS lacked exchangespliting along the spin channels at $\mathrm{E}_{\mathrm{F}}$. Magnetic half-metals are required for spintronic applications. The band structure along the high symmetry points for the up-spin and downspin channels are shown in Figs. $3 \& 4$. All the four alloys alloys showed zero energy band gap (characteristic of metals) in their up-spin channels (see Figs. 3(b), 3(d), 4(b) and 4(d)), while only $\mathrm{NbRhGeFe}$ and $\mathrm{NbRhGeCo}$ showed indirect energy band gap (characteristic of a semiconductor) for the down-spin channels (see Figs. 3 (a) \& (c)). The GGA predicted band gap for $\mathrm{NbRhGeFe}$ was $0.301 \mathrm{eV}$ and 0.644 $\mathrm{eV}$ for $\mathrm{NbRhGeCo.}$

\section{Phonon Dispersion}

The phonon dispersion provides a valid tool to evaluate structural stability for a system. The calculated phonon curve along high symmetry directions in the Brillouin zone for NbRhGeX ( $\mathrm{X}=\mathrm{Fe}, \mathrm{Co}, \mathrm{Ni}, \mathrm{Cr}$ ) are shown in Fig. 5 (a) through (d). A structurally stable structure is obtained when the frequency of all phonons across Brillouin zone are positive at absolute zero temperature. Otherwise, the structure is unstable [52]. It can be observed that no imaginary vibration frequency appeared for $\mathrm{NbRhGeCo}$, $\mathrm{NbRhGeFe}$ and $\mathrm{NbRhGeCr}$ while there was for $\mathrm{NbRhGeNi}$. This suggests that $\mathrm{NbRhGeFe,} \mathrm{NbRhGeCo}$ and $\mathrm{NbRhGeCr}$ are dynamically stable at absolute temperature.

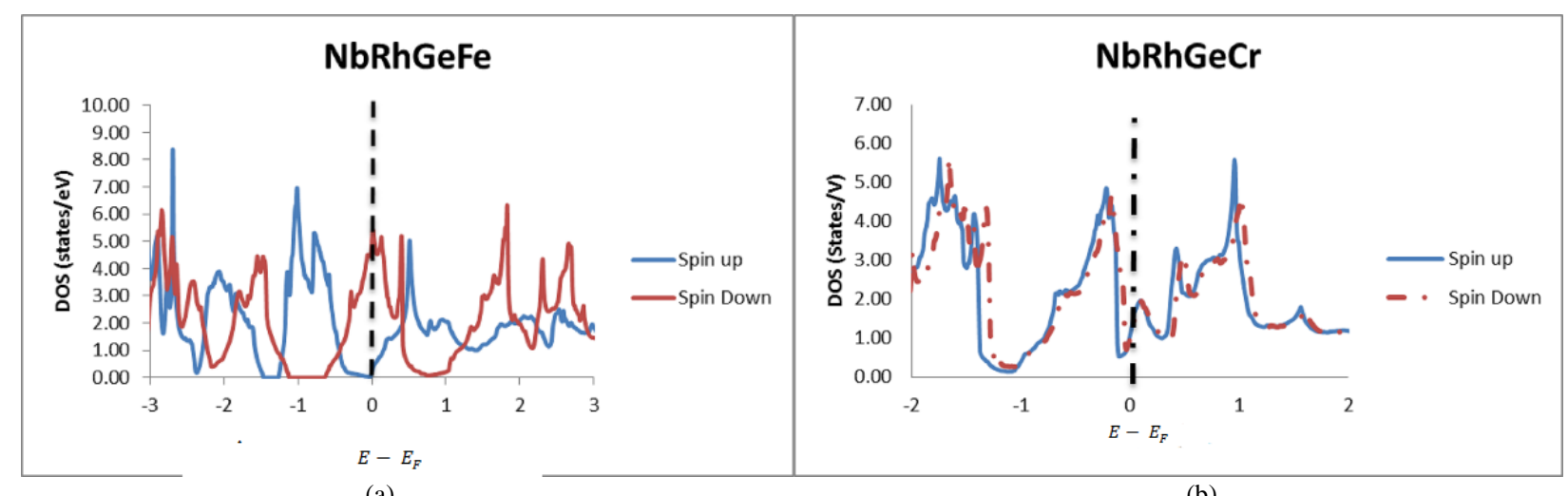

(a)

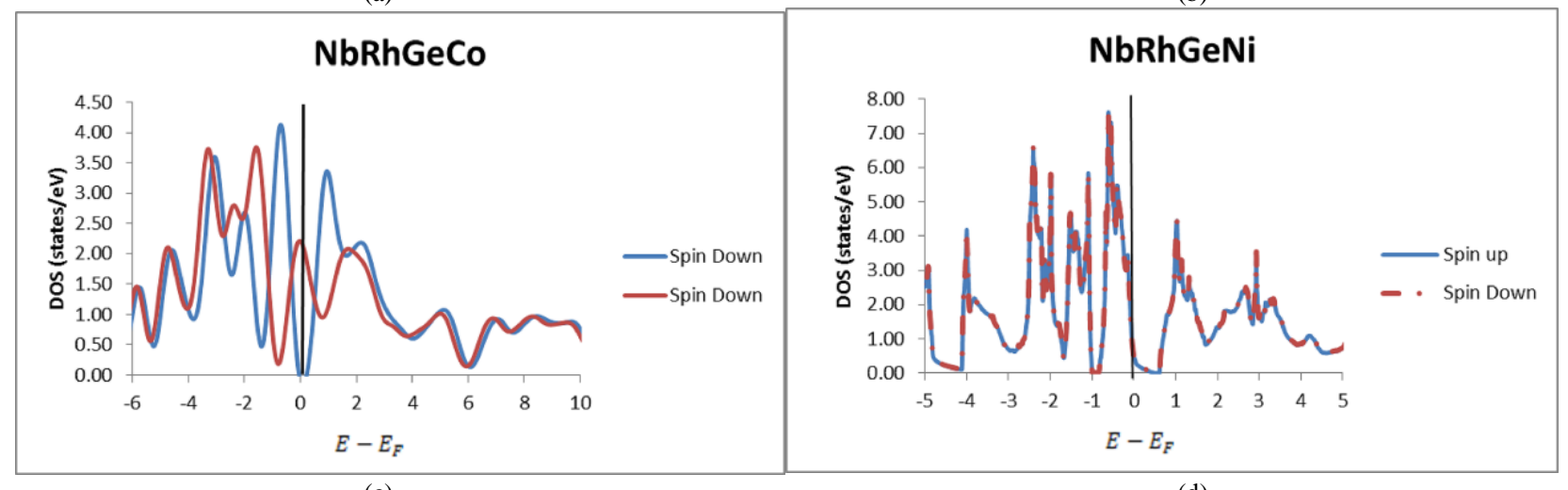

(c)

Fig. 2. Calculated Density of states plot for $\mathrm{NbRhGeX}(\mathrm{X}=\mathrm{Fe}, \mathrm{Cr}, \mathrm{Co}, \mathrm{Ni})$. 

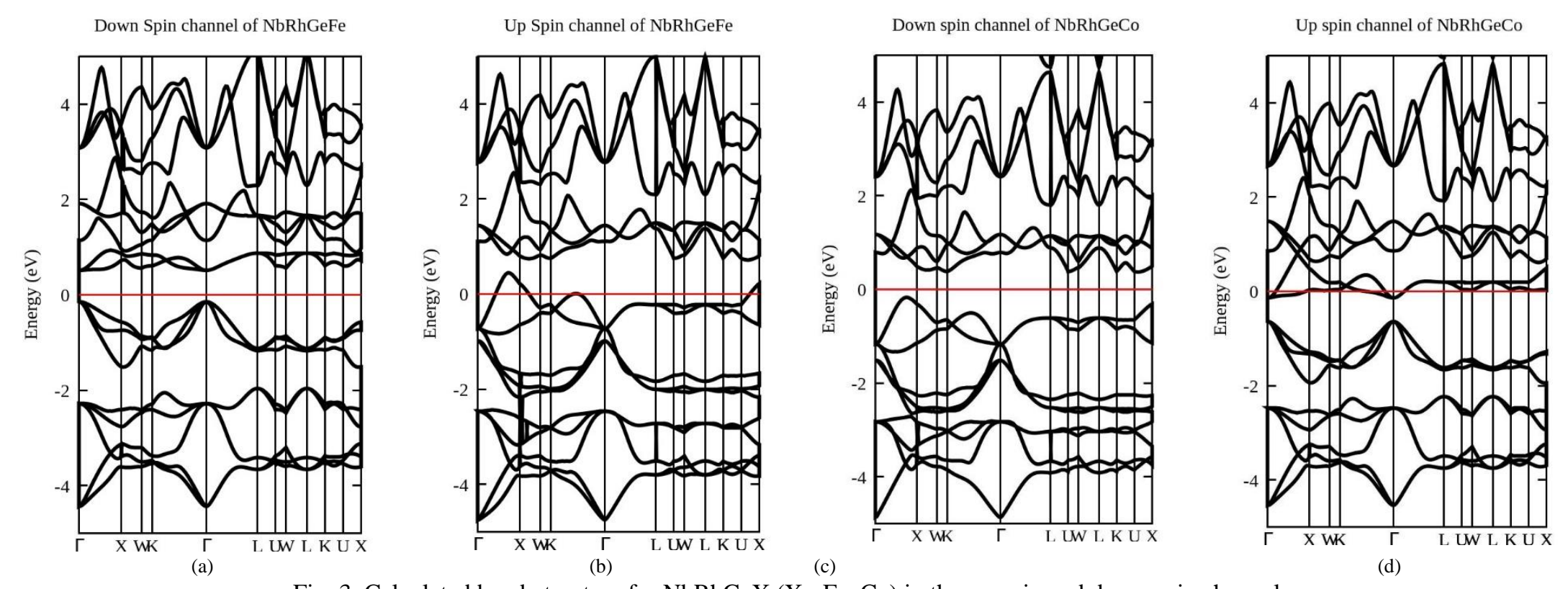

Fig. 3. Calculated band-structure for $\mathrm{NbRhGeX}(\mathrm{X}=\mathrm{Fe}, \mathrm{Co})$ in the up-spin and down-spin channels.
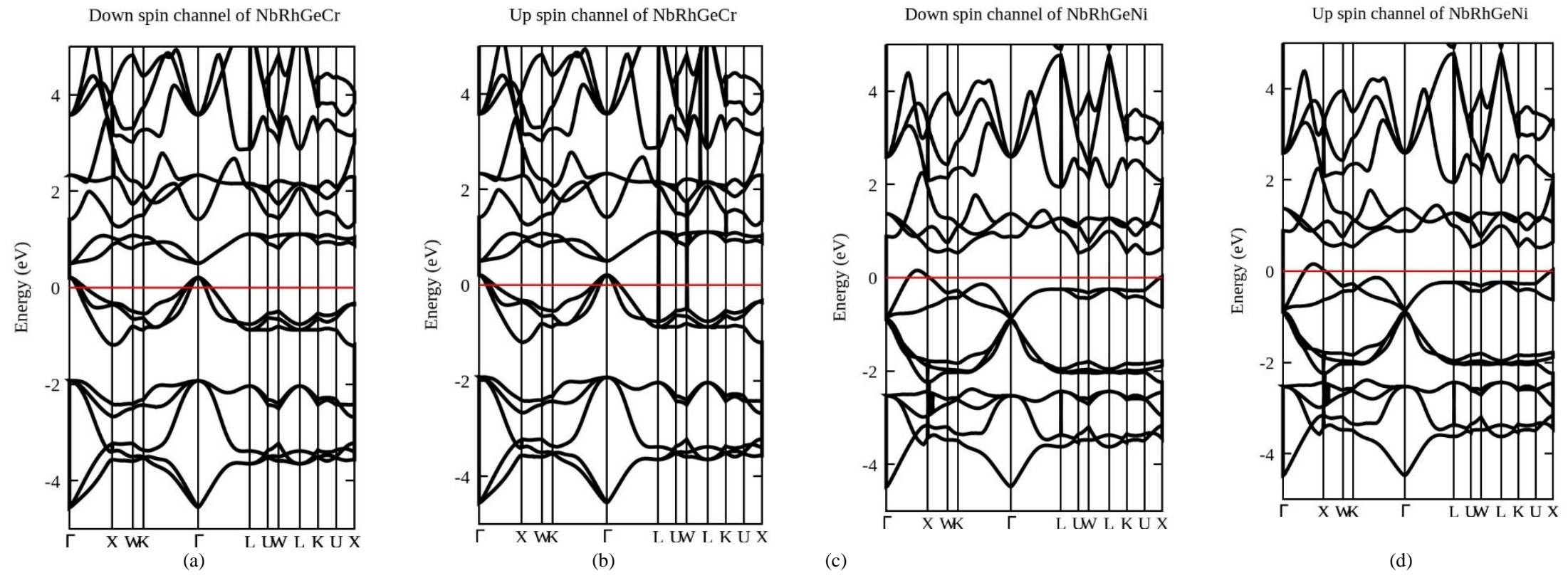

Fig. 4. Calculated band-structure for $\mathrm{NbRhGeX}(\mathrm{X}=\mathrm{Cr}, \mathrm{Ni})$ in the up-spin and down-spin channels. 
phonon dispersion of NbRhGeCr
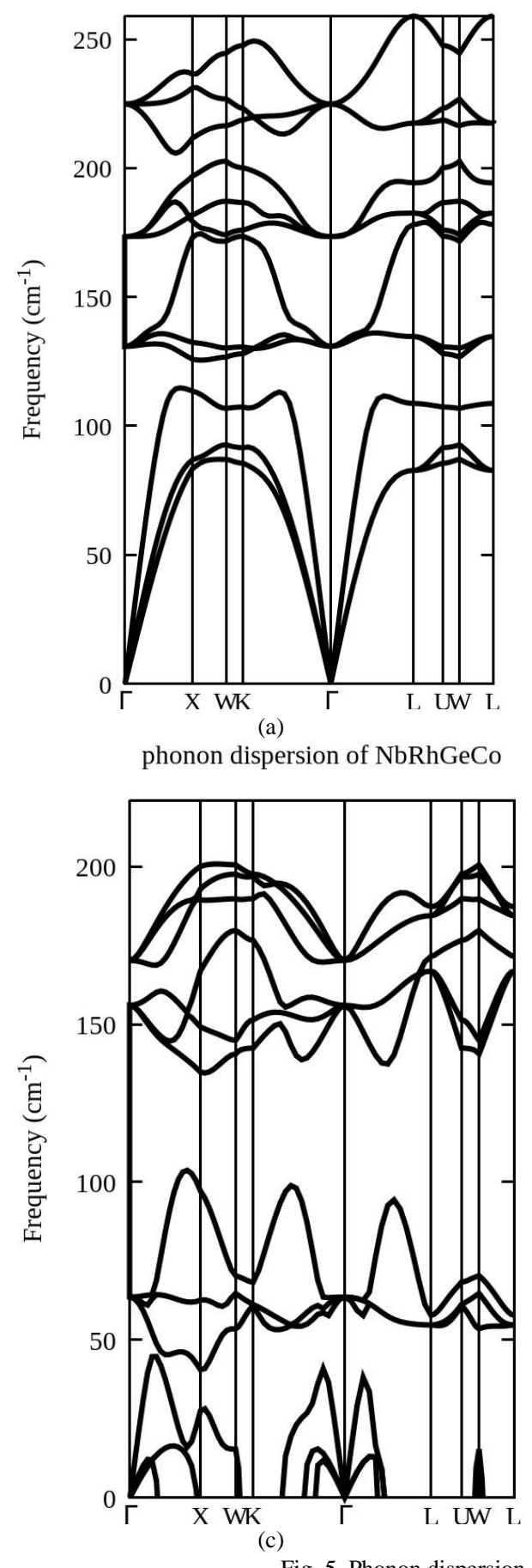

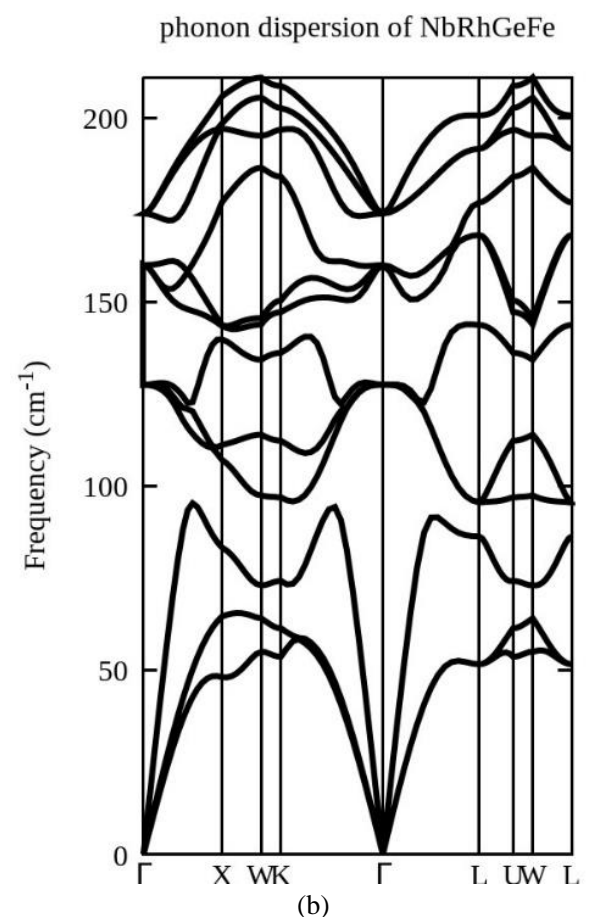

phonon dispersion curve of NbRhGeNi

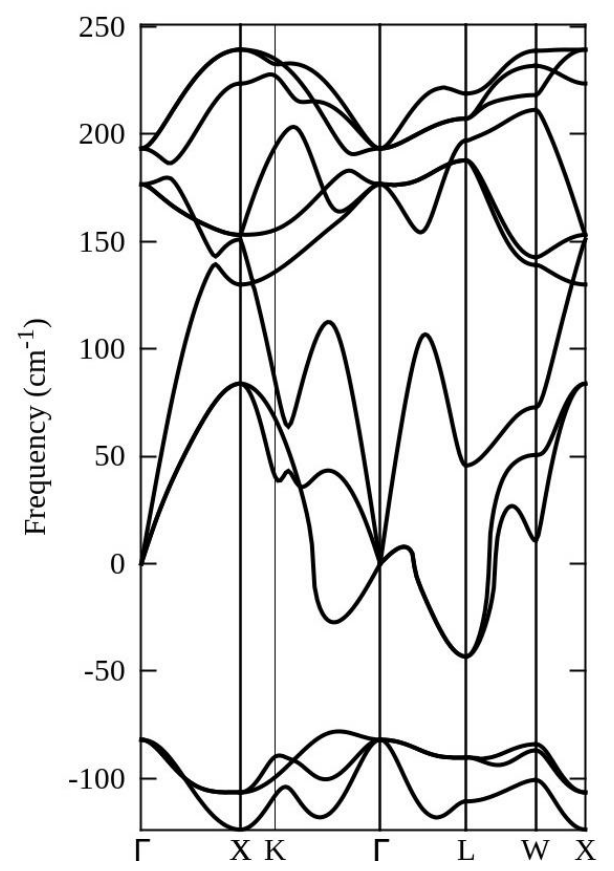

(d)

\section{CONCLUSION}

Any data storage media that utilizes the spintronic application is expected to be a magnetic component with $100 \%$ polarization. Four quaternary alloys have been investigated for possible use in spintronics. The electronic, magnetic, mechanical, and structural properties of

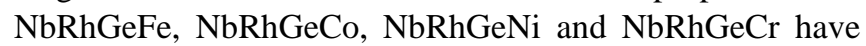
been evaluated. The electronic structure showed that only $\mathrm{NbRhGeFe}$ and $\mathrm{NbRhGeCo}$ are magnetic with the required $100 \%$ polarization. The other allied properties such as structure and mechanical stability was satisfied by $\mathrm{NbRhGeFe}$ while $\mathrm{NbRhGeCo}$ is unfortunately structurally and mechanically unstable. Conclusively and as summarized in Table II, NbRhGeFe is predicted suitable for spintronic application while $\mathrm{NbRhGeCr}$ can be considered for use in other application areas but certainly not for spintronics. $\mathrm{NbRhGeCo}$ and $\mathrm{NbRhGeNi}$ are unstable compositions which does not deserve further attention. $\mathrm{NbRhGeCr}$ and $\mathrm{NbRhGeFe}$ are suggested for further studies especially at elevated temperature and pressure.

TABLE II: CRITERIA FOR SPINTRONIC APPLICATION AND HOW THE ALLOYS MEET THE REQUIREMENTS

\begin{tabular}{ccccc}
\multicolumn{5}{c}{ ALLOYS MEET THE REQUIREMENTS } \\
\hline \multirow{2}{*}{ Compound } & $\begin{array}{c}100 \% \\
\text { Polarization }\end{array}$ & $\begin{array}{c}\text { Mechanical } \\
\text { Stability }\end{array}$ & $\begin{array}{c}\text { Structure } \\
\text { Stability }\end{array}$ & $\begin{array}{c}\text { Magnetic } \\
\text { Moment }\end{array}$ \\
\hline NbRhGeCr & $\mathbf{x}$ & $\checkmark$ & $\checkmark$ & x \\
NbRhGeCo & $\checkmark$ & $\mathbf{x}$ & $\mathbf{x}$ & $\checkmark$ \\
NbRhGeFe & $\checkmark$ & $\checkmark$ & $\checkmark$ & $\checkmark$ \\
NbRhGeNi & x & x & x & x \\
\hline
\end{tabular}




\section{ACKNOWLEDGMENT}

The authors would like to thank the Department of Physics of the Federal University of Technology Akure (FUTA) for providing the back-up power supply during the computations.

\section{REFERENCES}

[1] H. Sato, M. Yamanouchi, K. Miura, S. Ikeda, H. D. Gan, K. Mizunuma, R. Koizumi, F. Matsukura, and H. Ohno, Appl. Phys. Lett. 99, 042501 (2011).

[2] K. Song, S. C. Lee, and K. J. Lee, IEEE Trans. Magn. 50, 3400704 (2014).

[3] N.S. Kim et al., "Leakage current: Moore's law meets the static power", Computer, vol. 36, pp. 68-75, 2003.

[4] C. Chappert, A. Fert and F. Nguyen Van Dau, "The emergence of spin electronics in data storage", Nat. Mater., vol. 6, pp. 813-823, 2007.

[5] C.J. Lin et al., " $45 \mathrm{~nm}$ Low power CMOS logic compatible embedded STT MRAM utilizing a reverse-connection 1T/1MTJ cell”, Procs. Of IEDM, pp. 279-282, 2009.

[6] K. Tsuchida et al., "A $64 \mathrm{Mb}$ MRAM with clamped-reference and adequate-reference schemes", Procs. of ISSCC, pp. 258-259, 2010.

[7] Groot, R.A.D.; Mueller, F.M.; Engen, P.G.V.; Buschow, K.H.J. New class of materials: Half-metallic ferromagnets.Phys. Rev. Lett. 1983, $50,2024-2027$.

[8] de Groot,RA.Half-metallic magnetism in the 1990s. Physica B1991; 172:45-50

[9] Zhang,Z and Satpathy,S. Electron states, magnetism, and the Verwey transition in magnetite.Phys Rev B1991; 44:13319-31.

[10] van Leuken,Hand de Groot,RA.Half-metallic antiferromagnets. Phys Rev Letter 1995;74:1171-3.

[11] Dedkov, YS, Rüdiger, U and Güntherodt,G. Evidence for the halfmetallic ferromagnetic state of $\mathrm{Fe} 3 \mathrm{O} 4$ by spin-resolved photoelectron spectroscopy. Phys Rev B 2002; 65:064417.

[12] Kane, CLand Mele, EJ. Z2topological order and the quantum spin Hall effect. Phys Rev Lett 2005; 95:146802.

[13] König,M,Wiedmann, S and Brüne, C et al.Quantum spin Hall insulator state in huge quantum wells. Science 2007; 318:766-70

[14] Wang XL. Proposal for a new class of materials: spin gapless semiconductors. Phys Rev Lett 2008; 100: 156404.

[15] Wang XL, Dou SX and Zhang C. Zero-gap materials for future spintronics, electronics and optics. NPG Asia Mater 2010; 2: 31-8.

[16] Mak KF, Lee $\mathrm{C}$ and Hone $\mathrm{J}$ et al. Atomically thin MoS2: a new directgap semiconductor. Phys Rev Lett 2010; 105: 136805.

[17] Deng, Z, Jin,CQ and Liu, QQ et al.Li(Zn,Mn)As as a new generation ferromagnet based on a I-II-V semiconductor. Nat Commun 2011; 2:422.

[18] Li XX, Wu, XJ and Li, ZY et al. Proposal of a general scheme to obtain room-temperature spin polarization in asymmetric antiferromagnetic semiconductors.Phys RevB2015;92:125202.

[19] $\mathrm{Li}, \mathrm{XX}, \mathrm{Wu}, \mathrm{XJ}$ and $\mathrm{Li}, \mathrm{ZY}$ et al.Bipolar magnetic semiconductors: a new class of spintronics materials. Nanoscale 2012; 4:5680-5.

[20] Huang, PR, He, Y and Pal, HK et al. Prediction of switchable half semiconductor in d1transition metal dichalcogenidemonolayers.arXiv preprint 2015 , arXiv: 1501.00760

[21] Datta, Das, Appl. Phys. Lett. 56 (1990) 665

[22] Gregor, F.; Perter, K. Ternary semiconductors NiZrSn and CoZrBi with half-Heusler structure: A first-principles study. Phys. Rev. B. 2016, 94, 075203.

[23] Wang, X.T.; Cheng, Z.X.; Wang, W.H. L21 and XA Ordering Competition in Hafnium-Based Full-Heusler Alloys Hf2VZ (Z = Al, $\mathrm{Ga}, \mathrm{In}, \mathrm{Tl}, \mathrm{Si}, \mathrm{Ge}, \mathrm{Sn}, \mathrm{Pb})$. Materials 2017, 10, 1200. [CrossRef].

[24] Barman, C.K.; Alam, A. Topological phase transition in the ternary half-Heusler alloy ZrIrBi. Phys. Rev. B.2018, 97, 075302.

[25] Popoola, A. \& Odusote, Yisau. (2019). The Properties of NbRhGe as High Temperature Thermoelectric Material. IOSR Journal of Applied Physics. 11. 51-56. 10.9790/4861 1104025156.

[26] Giannozzi P., Baroni S., Bonini N., Calandra M., Car R., Cavazzoni C. et al. (2009) QUANTUMESPRESSO: a modular and open-source software project for quantum simulations of materials. J. Phys. Condens. Matter.vol21 no. 39, pp. 395502.

[27] Kohn, W., \& Sham, L.J. (1965). Self-consistent equations including exchange and correlation effects. Physical Reviews A, 140, 11331138.

[28] Perdew J. P., Burke K and Ernzerhof M. (1996) Generalized Gradient Approximation Made Simple. Phys. Rev. Lett.vol77 no.7, pp. 3865.

[29] Monkhorst H. J and Pack J. D. (1976) Special points for Brillouin-zone integrations. Phys. Rev. B. vol 13 no. 12, pp. 5188.
[30] Murnaghan F.D. (1944) The Compressibility of Media under Extreme Pressures. Proc. Natl. Acad. Sci. U. S. A. vol 30 no. 9, pp. 244-247.

[31] Papaconstantopoulos, D.A., \& Mehl, M.J. (2005). Tight Binding Method in Electronic Structure. Encyclopedia of Condensed Matter Physics, Elsevier B.V.

[32] Hill, R. (1952). The elastic behavior of a crystalline aggregate Proceedings of the Physical Society, 65, 349 -354.

[33] Zener, C. (1948). Elasticity and inelasticity of metals. University of Chicago Press.

[34] Güler, E., \& Güler, M. (2014). Phase transition and elasticity of gallium arsenide under pressure. Materials Research, 17(5), 1268-1272.

[35] Chen, X.Q., Niu, H., Li, D., \& Li, Y. (2011). Modeling hardness of polycrystalline materials and bulk metallic glasses. Intermetallic, 19(9), 1275-1281.

[36] Anderson, O. L. (1963). A simplified method for calculating the Debye temperature from elastic Constants. Journal of Physics and Chemistry of Solids, 24(7), 909-917.

[37] Togo, A.; Tanaka, I. First principles phonon calculations in materials science. Scr. Mater. 2015, 108, 1

[38] Born, M. (1940). On the stability of crystal lattices. Mathematical Proceedings of the Cambridge Philosophical Society, 36, 160-172.

[39] Bensalem S, Chegaar M, Maouche D and Bouhemadou A. Theoretical study of structural, elastic and thermodynamic properties of CZTX (X $=\mathrm{S}$ and Se) alloys. Journal of Alloys and Compounds. 2014; 589:137 142. http://dx.doi.org/10.1016/j. jallcom.2013.11.113.

[40] Tariq, S., Ahmed, A., Saad, S., \& Tariq, S. (2015). Structural, electronic and elastic properties of the cubic $\mathrm{CaTiO}$ under pressure: A DFT study. AIP Advances, 5 (7), 077111.

[41] Kanchana V and Ram S. Electronic structure and mechanical properties of $\operatorname{Sc} 3 \mathrm{AC}(\mathrm{A}=\mathrm{Al}, \mathrm{Ga}, \mathrm{In}, \mathrm{Tl})$ and $\mathrm{Sc} 3 \mathrm{BN}(\mathrm{B}=\mathrm{Al}, \mathrm{In})$ : Ab-initio study. Intermetallics. $2012 ; \quad 23: 39-48$ http://dx.doi.org/10.1016/j.intermet.2011.12.014.

[42] Ustundag M, Aslan M and Yalcin BG. The first-principles study on physical properties and phase stability of Boron-V (BN, BP, BAs, BSb and $\mathrm{BBi}$ ) compounds. Computational Materials Science. 2014; 81:471477. http://dx.doi.org/10.1016/j. commatsci.2013.08.056.

[43] Liu Z.T.Y, Gall D and Khare S.V. (2014). Electronic and bonding analysis of hardness in pyrite-typetransition-metal pernitrides. Phys. Rev. B, vol 90 no. 13, pp. 134102.

[44] Bensalem S, Chegaar M, Maouche D and Bouhemadou A. Theoretical study of structural, elastic and thermodynamic properties of CZTX (X $=\mathrm{S}$ and Se) alloys. Journal of Alloys and Compounds. 2014; 589:137 142. http://dx.doi.org/10.1016/j. jallcom.2013.11.113.

[45] Guemou M, Abdiche A, Riane R and Khenata R. Ab initio study of the structural, electronic and optical properties of BAs and BN compounds and BNxAs1-x alloys. Physica B: Condensed Matter. 2014; 436:3340. http://dx.doi.org/10.1016/j. physb.2013.11.030.

[46] Gao X, Jiang Y, Zhou R and Feng J. Stability and elastic properties of $\mathrm{Y}-\mathrm{C}$ binary compounds investigated by first principles calculations. Journal of Alloys and Compounds. 2014; 587:819-826. http://dx.doi.org/10.1016/j.jallcom.2013.11.005.

[47] Zhang M, Yan H, Zhao Y and Wei Q. Mechanical properties and atomistic deformation mechanism of spinel-type BeP2N4 Computational Materials Science. 2014; 83:457-462 http://dx.doi.org/10.1016/j.commatsci.2013.11.044.

[48] Wang S, Li JX, Du YL and Cui C. First-principles study on structural, electronic and elastic properties of graphenelike hexagonal Ti2C monolayer. Computational Materials Science. 2014; 83:290-293 http://dx.doi.org/10.1016/j. commatsci.2013.11.025.

[49] Feng LP, Li N, Yang MH and Liu ZT. Effect of pressure on elastic, mechanical and electronic properties of WSe2: a firstprinciples study. Materials Research Bulletin. 2014; 50:503508 http://dx.doi.org/10.1016/j.materresbull.2013.11.016

[50] Greaves GN, Greer AL, Lakes RS and Rouxel T. Poisson's ratio and modern materials. Nature Materials. 2011; 10:823-837. PMid:22020006. http://dx.doi.org/10.1038/nmat3134.

[51] Güler $M$ and Güler E. Embedded atom method-based geometry optimization aspects of body-centered cubic metals. Chinese Physics Letters. 2013; 30(5):056201. http://dx.doi.org/10.1088/0256307X/30/5/056201.

[52] Chen, Q.; Wang, J.L. Structural, electronic, and magnetic properties of TMZn11O12 and TM2Zn10O12 clusters $(\mathrm{TM}=\mathrm{Sc}, \mathrm{Ti}, \mathrm{V}, \mathrm{Cr}, \mathrm{Mn}, \mathrm{Fe}$, Co, Ni, and Cu). Chem. Phys. Lett. 2009, 474, 336-341.

A. I. Popoola is a faculty member at the Federal University of Technology, Akure, Ondo State, Nigeria. He is currently an Associate Professor. His research centers on electronics and materials modeling.

S. B. Akinpelu is a graduate student under the advice of AIP. His research work focusses on spintronic and thermo-electric materials. 